\title{
Validation of respiratory rate measurements from remote monitoring device in COPD patients
}

J. Soler ${ }^{a, b}$, J. Alves Pegoraro ${ }^{a, c, d}$, X. L. Le ${ }^{c}$, D. P. Q. Nguyen ${ }^{c}$, L. Grassion ${ }^{a, e}$, R. Antoine $^{a}$, A. Guerder ${ }^{a}$, J. Gonzalez-Bermejo ${ }^{a, f}$

a. AP-HP, Groupe Hospitalier Pitié-Salpetrière Charles Foix, Service de Pneumologie et Réanimation Médicale (Département "R3S"), 47-83 Boulevard de l'Hôpital, 75013, Paris, France

b. CHRU de Nancy, Département de Pneumologie, Rue du Morvan, 54500, Vandoeuvre-lès-Nancy, France

c. SRETT, 305 avenue Le Jour se Lève, 92100 Boulogne-Billancourt, France

d. Université Paris Descartes, UMR CNRS 8145, Laboratoire MAP5, 45 rue des Saints-Pères, 75006 Paris, France

e. Hôpital du Haut Lévèque, Avenue Magellan, Service des Maladies Respiratoires, 33604 Pessac, France

f. Sorbonne Université, UPMC Université Paris 06, INSERM, UMRS 1158 Neurophysiologie respiratoire expérimentale et clinique, 47-83 Boulevard de l'Hôpital, 75651 Paris Cedex 13, France

Corresponding Author: j.pegoraro@srett.com 
With healthcare objectives and budget constraint, remote monitoring of chronic obstructive pulmonary disease (COPD) patients is an important challenge in most European countries.[1] Recent works have shown that it is possible to predict COPD exacerbation based on monitoring of simple parameters, such as the respiratory rate (RR) of the patient in spontaneous ventilation [2-4] or under non-invasive ventilation [5]. Until now, these devices do not allow a daily automatic data remote transmission,[4] or it is restricted to patients under mechanical ventilation [5]. TeleOx $^{\circledR}$ (SRETT, Boulogne-Billancourt, France), the first oxygen flow rate remote monitoring device, also allows a RR measurement by associating a pressure sensor and a fluidic oscillator flow sensor. A median RR is output every 5 minutes based on time interval between two consecutive respiratory cycles.

In this study, we compared the corresponding RR measurements between TeleOx ${ }^{\circledR}$ and the reference polygraph (Nox-T3 ${ }^{\circledR}$, Nox Medical Inc. Reykjavik, Iceland) from COPD patients under nasal oxygen therapy with flow rates between 0.5 and 5.0 litres per minute. Patients without mechanical ventilation and without any other respiratory pathology were eligible to the study if they would undergo a ventilatory polygraph record for other reasons.

This study was approved by our institutional board (CEPRO $n^{\circ} 2016-005$ ).

Result agreement was estimated using the Bland-Altman method, presented here, and the Passing-Bablok regression method, for non-parametric regression analysis, presented in the online supplement. 
In order to obtain an error margin of $3 \%$ and a confidence interval of $95 \%, 1000$ valid measurement points were necessary, which demands 14 patients' recordings of about 6 hours each.

Results are presented as mean \pm standard deviation.

Several software versions were necessary to improve its reliability. The first version of TeleOx ${ }^{\circledR}$ was used to record 8 patients, the second version 12 and the third version 14 patients with respectively $38 \%, 33 \%$ and $93 \%$ of success rate. Here, we analyse results from the last version.

From the fourteen patients included, 1099 valid measurement points allowed statistical analysis. Included patients exhibit a forced expiratory volume in one second (FEV1) of $1032 \pm 619 \mathrm{ml}$ (or $44 \pm 23 \%$ of predicted value), a PaO2 in spontaneous ventilation and ambient air at $65.3 \pm 6.9 \mathrm{mmHg}$, a PaCO2 at $43.8 \pm 5.1 \mathrm{mmHg}$. $21 \%$ of the population was classified GOLD B, 64\% GOLD D, 7\% GOLD A and 7\% GOLD C. Anthropometric data can be found in the online supplement.

The RR measures from the polygraph were $19.45 \pm 5.39$ breaths per minute and from the TeleOx ${ }^{\circledR}$ were $19.41 \pm 5.38$ breaths per minute.

The bias calculated by Bland-Altman method is 0.046 within $[-3.865,3.957]$ limits of agreement at $95 \%$ (figure 1).

No participant found TeleOx ${ }^{\circledR}$ intrusive. 


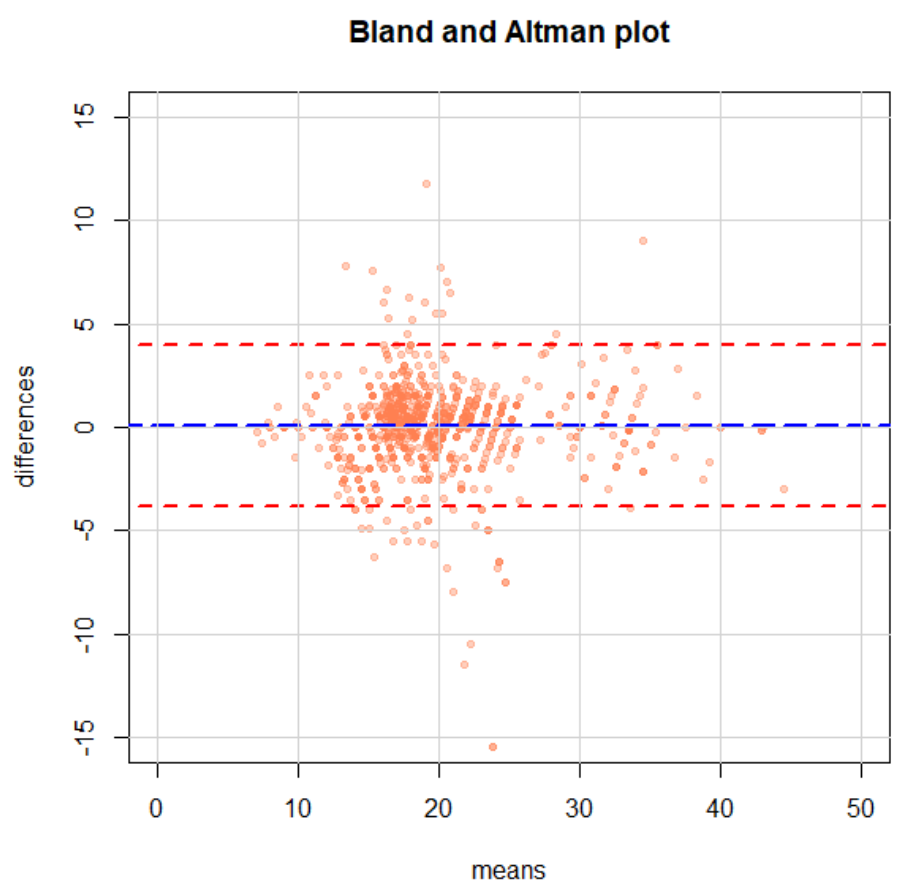

Figure 1:

Comparison using Bland-Altman method of respiratory rate (RR) measurements by TeleOx ${ }^{\circledast}$ and Nox-T3 on a set of 1099 valid measurement points. Bias is presented by a dashed blue line and upper and lower 95\% limits of agreement of the differences are presented by dashed red lines.

A recent study on $\mathrm{RR}$ remote monitoring devices shows a measure quality discrepancy, with measurement bias of 3.01 and -3.21 cycles/minute (corresponding limits of agreement are -11.17 to 17.19 and -12.71 to 6.30 ) for measurement methods based on photoplethysmography and on camera respectively.[6] Compared to these devices, the measurement bias of TeleOx ${ }^{\circledR}$ is far smaller and its limits of agreement interval is narrower. This device is also less intrusive and thus may improve patient adhesion to remote monitoring. 
Important RR measurement differences are found to happen in periods where artefacts (as apnoea, hypopnoea and mouth breathing) take place, which makes median RR more difficult to measure to both devices, or during the transition zone (first minutes of the recording when TeleOx ${ }^{\circledR}$ is calibrating).

No analysis considering each patient separately is presented because most of the patients present a narrow range of RR values combined with a small sample size, interfering method comparison. Moreover, we considered the addition of more patients unnecessary, since we have the aimed sample size of RR measurements covering a large range of RR values.

As it has been shown with VisionOx ${ }^{\circledR}$, another tool described in 2012 (finally not developed as such because it was not communicating), more and more evidence suggests that an increase in RR is predictive of an exacerbation.[4] In addition, a recent study demonstrated the benefit of monitoring RR by photoplethysmography using a pulse oximeter in the COPD patient homes. [7] Such monitoring is, however, dependent on the patient's compliance.

To the best of our knowledge, TeleOx is the first near real-time RR remote monitoring tool for COPD patient under oxygen therapy without mechanical ventilation. This wireless connected device is non-invasive and simple to use, allowing for remote monitoring of treatment compliance, oxygen flow rate and now the RR of the patient. These features make it an excellent tool for LTOT patient remote monitoring. The next step of our work is to test the ability to capture changes in RR of the patients during stable state, acute exacerbation and recovery. Future development will need to address the detection of small changes in breathing rate patterns in the context of a 
complex background.

\section{Declarations:}

Ethics approval and informed consent:

This study was approved by our institutional board (CEPRO $\left.n^{\circ} 2016-005\right)$, Consent to

participate was obtained

Consent for publication:

All the authors agreed to this publication

Data availability:

In supplementary material

Funding:

No funding was received

Conflict of interest:

X. L. Le and D. P. Q Nguyen are employed by SRETT.

J. Alves Pegoraro is member of the project PHD CIFRE from the French government and her salary is paid by SRETT.

No conflict of interest, financial or other, exists for the authors.

The abstract of this paper was presented at the British Thoracic Society Winter Meeting 2017 as a poster presentation with interim findings. The poster's abstract was published in "Poster Abstracts" in Thorax: http://dx.doi.org/10.1136/thoraxinl-2017210983.208

Authors' contributions:

J.G-B, X.L.L, J.S, R.A, A.G, L.G: conception, hypothesis delineation and design of the 
study. J.G-B, J.S, X.L.L, D.P.Q.N, L.G, A.G, J.A.P: Acquisition of data or analysis and interpretation. J.G-B, J.S, X.L.L, D.P.Q.N, L.G, R.N, A.G, J.A.P: Writing the article and correction

Acknowledgements:

We thank Pr Thomas Similowski (Groupe Hospitalier Pitié-Salpetrière Charles Foix, Service de Pneumologie et Réanimation Médicale, Paris) for advice on statistical analysis. In addition, we thank the paramedic staff of "R3S" department for the data collection.

\section{References}

[1] The Commission To The European Parliament, The Council, The European Economic And Social Committee And The Committee Of The Regions. eHealth Action Plan 20122020 - Innovative healthcare for the 21st century. Brussels, Belgium; 2012.

[2] Ballal T, Heneghan C, Zaffaroni A, Boyle P, de Chazal P, Shouldice R, et al. A pilot study of the nocturnal respiration rates in COPD patients in the home environment using a non-contact biomotion sensor. Physiol Meas. 2014;35:2513-27. https://doi.org/10.1088/0967-3334/35/12/2513

[3] Droitcour AD, Seto TB, Park B-K, Yamada S, Vergara A, El Hourani C, et al. Noncontact respiratory rate measurement validation for hospitalized patients. Eng Med Biol Soc. 2009 EMBC 2009 Annu. Int. Conf. https://doi.org/10.1109/IEMBS.2009.5332635 [4] Yañez AM, Guerrero D, de Alejo RP, Garcia-Rio F, Alvarez-Sala JL, Calle-Rubio M, et al. Monitoring breathing rate at home allows early identification of COPD 
exacerbations. CHEST J. 2012;142:1524-9. https://doi.org/10.1378/chest.11-2728

[5] Borel J-C, Pelletier J, Taleux N, Briault A, Arnol N, Pison C, et al. Parameters recorded by software of non-invasive ventilators predict COPD exacerbation: a proofof-concept study. Thorax. 2015;70:284-5. https://doi.org/10.1136/thoraxjnl-2014206569

[6] Rubio N, Parker RA, Drost EM, Pinnock H, Weir CJ, Hanley J, et al. Home monitoring of breathing rate in people with chronic obstructive pulmonary disease: observational study of feasibility, acceptability, and change after exacerbation. Int J Chron Obstruct Pulmon Dis. 2017;Volume 12:1221-31. https://doi.org/10.2147/COPD.S120706 [7] Shah SA, Velardo C, Farmer A, Tarassenko L. Exacerbations in Chronic Obstructive Pulmonary Disease: Identification and Prediction Using a Digital Health System. J Med Internet Res. 2017;19:e69. https://doi.org/10.2196/jmir.7207 